\title{
O Modelo da Esfera Rígida em Folha de CÁlculo: geometrias DE REPULSÃO MÍNIMA DA TETRA À NONACOORDENAÇÃO
}

\author{
Mário Valente \\ Colégio D. Duarte, Porto \\ madmage1@yahoo.com
}

Neste trabalho descreve-se a utilização do modelo da esfera rígida, em folha de cálculo, para determinação de geometrias moleculares de repulsão mínima, com o propósito de demonstrar a possibilidade de utilização de meios informáticos ubíquos, em conjunto com modelos teóricos simples, para a realização de modelações úteis em Química. Como resultado são descritas as geometrias ideais mais estáveis segundo este modelo, para moléculas do tipo $A B_{n}, \operatorname{com} n=4$ a 9 .

\section{INTRODUÇÃO}

Numa época em que a modelação computacional apresenta um interesse crescente nos mais variados campos, constituiu para nós um desafio pedagógico interessante a elaboração de uma aplicação informática que fosse simultaneamente um exemplo de modelação computacional aplicada à Química e fizesse uso de conceitos de Matemática e Física suficientemente simples para poderem ser entendidos por alunos dos últimos anos do ensino secundário. Nos programas da disciplina de Física e Química A (níveis 1 e 2) [1] mencionam-se, na sua componente de Química, geometrias moleculares simples, bem como na sua componente de Física, campo eléctrico e atracção / repulsão electrostática: a base teórica deste modelo.

Como ferramenta de trabalho considerou-se a utilização de uma folha de cálculo, já que este instrumento é hoje acessível de forma praticamente ubíqua.

Neste artigo relatam-se os resultados obtidos para a determinação das geometrias de repulsão mínima até à nonacoordenação, portanto bem além do limite da hexacoordenação considerado ao nível da disciplina opcional de Química do $12 .^{\circ}$ ano, e mais além ainda do limite da tetracoordenação, considerado na disciplina de Física e Química A.

É importante notar que para espécies moleculares nas quais um átomo central está rodeado por quatro a seis átomos periféricos, cada número de coordenação específico apresenta geralmente uma ou duas geometrias bastante mais frequentes do que outras possíveis. Para números de coordenação superiores a seis aumenta o número de geometrias de repulsão mínima já que as diferenças energéticas entre várias geometrias possíveis se esbatem, permitindo que, à temperatura ambiente, as referidas espécies moleculares assumam uma de várias geometrias possíveis ou até que apresentem geometrias fluxionais [2].

\section{DESCRIÇÃO DO MODELO}

Como base teórica usou-se o Modelo da Esfera Rígida que se baseia na distribuição de um dado número de cargas pontuais (representando os átomos periféricos) à superfície de uma esfera em cujo centro se encontra o átomo central. De seguida a posição das cargas à superfície da esfera é variada por forma a minimizar a repulsão total entre elas.

Usando uma folha de cálculo (Microsoft ${ }^{\circledR}$ Office Excel 2003) criou-se uma tabela contendo as coordenadas esféricas angulares $(\theta, \varphi)$ de cada uma das cargas pontuais distribuídas numa geometria de coordenação próxima da desejada. É evidente que se torna desnecessário definir especificamente a coordenada esférica $r$, já que o seu valor é fixo (aqui tomado como unitário) e igual para todas as cargas pontuais - desta aproximação deriva o nome do modelo utilizado.

Numa outra tabela as coordenadas esféricas angulares foram convertidas em coordenadas cartesianas $(X, Y, Z)$, segundo:

$(\operatorname{sen}(\theta) \cdot \cos (\varphi), \operatorname{sen}(\theta) \cdot \operatorname{sen}(\varphi), \cos (\theta)) \rightarrow(X, Y, Z) \quad($ Eq. 1$)$

O cálculo da repulsão para cada par de cargas pontuais, $R_{a, b}$, foi efectuado segundo:

$R_{1,2}=\left(\left(x_{2}-x_{1}\right)^{2}+\left(y_{2}-y_{1}\right)^{2}+\left(z_{2}-z_{1}\right)^{2}\right)^{-\epsilon}$

em que $\left(x_{1}, y_{1}, z_{1}\right)$ e $\left(x_{2}, y_{2}, z_{2}\right)$ são as coordenadas cartesianas das cargas pontuais $\mathbf{1}$ e 2 , respectivamente. Em vez do expoente -2 (para uma repulsão puramente electrostática) usou-se o expoente -6 por ser considerado consensualmente [3-6] como o que apresenta resultados mais próximos dos experimentais.

A repulsão total, $R_{T}$, foi calculada como a soma de todas as repulsões entre os pares de um total de $n$ cargas pontuais:

$R_{T}=\sum_{i=1}^{n-1} \sum_{j=i=1}^{n} R_{i j}$

As geometrias moleculares de repulsão mínima, assumidas como sendo as mais estáveis, foram então determinadas fazendo variar gradualmente as coordenadas angulares de 
cada uma das cargas pontuais (isoladamente ou em grupo) até se atingir um mínimo local do valor de $R_{T}$ (ver Nota 1 ).

A visualização das geometrias resultantes dos cálculos foi realizada usando a versão experimental (gratuita) do programa ChemCraft [7]. Bastando para tal copiar as coordenadas cartesianas dos átomos para o Bloco de Notas do Windows (Microsoft ${ }^{\circledR}$ ) e construir com elas um ficheiro em que cada linha corresponde a um átomo (o central ou um dos periféricos) e contém o número atómico do átomo seguido das suas coordenadas $X, Y$ e $Z$. Cada valor numérico deve ser separado dos outros por um espaço (ver Nota 2). Depois de construído, o ficheiro deve ser gravado com a terminação $\mathbf{x y z}$ por forma a ser aceite pelo programa de visualização como um ficheiro de coordenadas cartesianas.

Apesar de o procedimento adotado fornecer resultados pedagogicamente interessantes considera-se fundamental salvaguardar que o modelo usado é muito simplista e que, na realidade, a adopção, por parte de uma molécula, de uma dada geometria resulta da conjugação de vários factores de complexidade consideravelmente elevada e, sob o ponto de vista teórico, muito além do que é possível abordar ao nível do ensino secundário.

\section{GEOMETRIAS IDEAIS PARA TETRACOORDENAÇÃO}

Para este número de coordenação as geometrias estudadas foram a tetraédrica (simetria $T_{d}$, Figura 1A), de menor valor de $R_{T}$ (Tabela 1), e a quadrada plana (simetria $D_{4 h}$, Figura 1B).
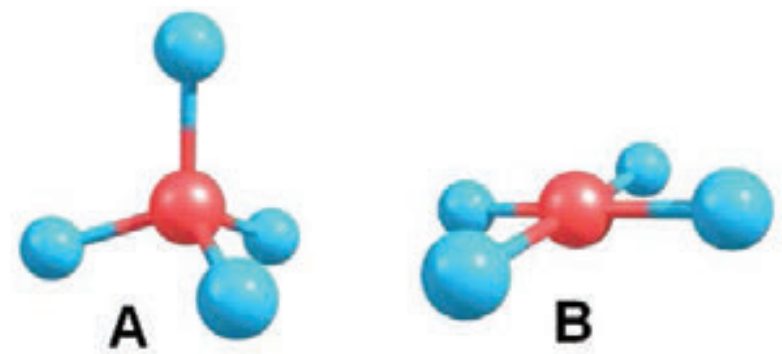

Figura 1 - Duas geometrias de menor valor de $R_{T}$ para espécies do tipo $\mathrm{AB}_{4}$

Tabela 1 - Valores de $R_{T}$ e coordenadas angulares usadas para os átomos periféricos referentes às duas geometrias mais estáveis para espécies do tipo $\mathrm{AB}_{4}$

\begin{tabular}{ccccc}
\hline & \multicolumn{2}{c}{$T_{d}$} & \multicolumn{2}{c}{$D_{4 h}$} \\
\hline$R_{T}$ & \multicolumn{2}{c}{0,01669} & \multicolumn{2}{c}{0,06299} \\
\hline & $\theta$ & $\varphi$ & $\theta$ & $\varphi$ \\
\hline 1 & $0^{\circ}$ & $0^{\circ}$ & $90^{\circ}$ & $0^{\circ}$ \\
\hline 2 & $109,5^{\circ}$ & $0^{\circ}$ & $90^{\circ}$ & $90^{\circ}$ \\
\hline 3 & $109,5^{\circ}$ & $120^{\circ}$ & $90^{\circ}$ & $180^{\circ}$ \\
\hline 4 & $109,5^{\circ}$ & $240^{\circ}$ & $90^{\circ}$ & $270^{\circ}$ \\
\hline
\end{tabular}

\section{GEOMETRIAS IDEAIS PARA PENTACOORDENAÇÃO}

No caso da pentacoordenação as geometrias moleculares estudadas foram a bipiramidal trigonal (simetria $D_{2 h}$, Fi- gura 2A), de valor de $R_{T}$ ligeiramente mais baixo (Tabela 2), e a piramidal quadrada (simetria $C_{4 v}$, Figura 2B).
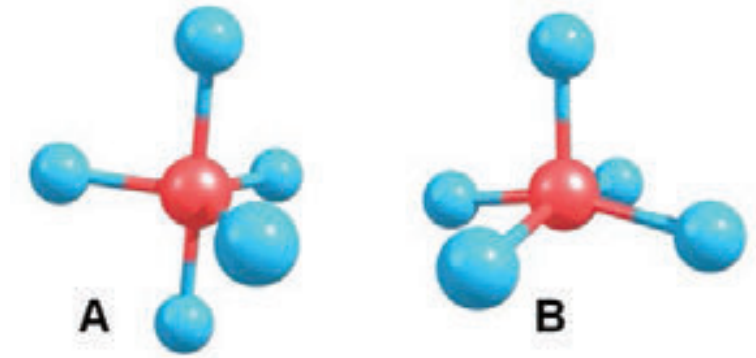

Figura 2 - Duas geometrias de menor valor de $R_{T}$ para espécies do tipo $A B_{5}$

Tabela 2 - Valores de $R_{T}$ e coordenadas angulares usadas para os átomos periféricos referentes às duas geometrias mais estáveis para espécies do tipo $A B_{5}$

\begin{tabular}{ccccc}
\hline & \multicolumn{2}{c}{$D_{3 h}$} & \multicolumn{2}{c}{$C_{4 v}$} \\
\hline$R_{T}$ & \multicolumn{2}{c}{0,09811} & \multicolumn{2}{c}{0,09921} \\
\hline & $\theta$ & $\theta$ & $\theta$ & $\varphi$ \\
\hline 1 & $0^{\circ}$ & $0^{\circ}$ & $98,8^{\circ}$ & $0^{\circ}$ \\
\hline 2 & $180^{\circ}$ & $180^{\circ}$ & $98,8^{\circ}$ & $0^{\circ}$ \\
\hline 3 & $90^{\circ}$ & $90^{\circ}$ & $98,8^{\circ}$ & $90^{\circ}$ \\
\hline 4 & $90^{\circ}$ & $90^{\circ}$ & $98,8^{\circ}$ & $180^{\circ}$ \\
\hline 5 & $90^{\circ}$ & $90^{\circ}$ & $98,8^{\circ}$ & $270^{\circ}$ \\
\hline
\end{tabular}

\section{GEOMETRIA IDEAL PARA HEXACOORDENAÇÃO}

A geometria molecular de menor valor de $R_{T}(0,1188)$ para a hexacoordenação é a octaédrica (simetria $O_{h}$, Figura 3).

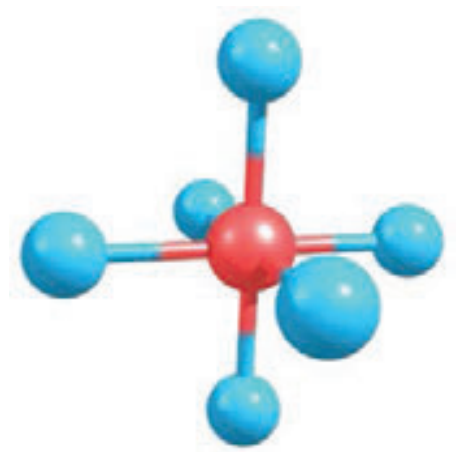

Figura 3 - A geometria de menor valor de $R_{T}$ para espécies do tipo $A B_{6}$

Tabela 3 - Valores de $R_{T}$ e coordenadas angulares usadas para os átomos periféricos referentes à geometria mais estável para espécies do tipo $A B_{6}$

\begin{tabular}{ccc}
\hline & \multicolumn{2}{c}{$O_{h}$} \\
\hline$R_{T}$ & \multicolumn{2}{c}{0,1882} \\
\hline & $\theta$ & $\varphi$ \\
\hline 1 & $0^{\circ}$ & $0^{\circ}$ \\
\hline 2 & $180^{\circ}$ & $0^{\circ}$ \\
\hline 3 & $90^{\circ}$ & $0^{\circ}$ \\
\hline 4 & $90^{\circ}$ & $90^{\circ}$ \\
\hline 5 & $90^{\circ}$ & $180^{\circ}$ \\
\hline 6 & $90^{\circ}$ & $270^{\circ}$ \\
\hline
\end{tabular}




\section{GEOMETRIAS IDEAIS PARA HEPTACOORDENAÇ̃̃O}

Verificou-se que a geometria molecular ideal com valor de $R_{T}$ mais baixo (Tabela 4) é a octaédrica de face "en capada" (simetria $C_{3 v}$, figura $4 \mathrm{~A}$ ). Contudo, duas outras geometrias moleculares ideais apresentam valores muito próximos do mínimo: a prismática de face "encapada" (simetria $C_{2 v}$, Figura 4B) e a bipiramidal pentagonal (simetria $D_{5 h}$, Figura 4 C).

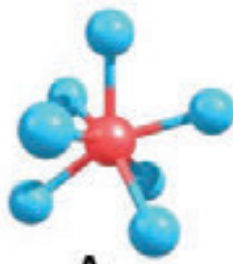

A

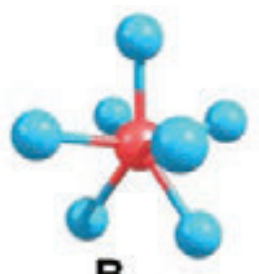

B

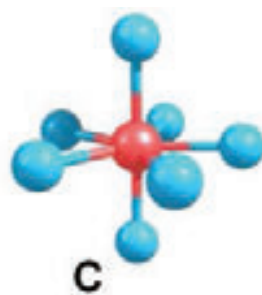

C
Figura 4 - Três geometrias de menor valor de $R_{T}$ para espécies do tipo $A B_{7}$

Tabela 4 - Valores de $R_{T}$ e coordenadas angulares usadas para os átomos periféricos referentes às três geometrias mais estáveis para espécies do tipo $\mathrm{AB}_{7}$

\begin{tabular}{ccccccc}
\hline & \multicolumn{2}{c}{$C_{3 v}$} & \multicolumn{2}{c}{$C_{2 v}$} & \multicolumn{2}{c}{$D_{5 h}$} \\
\hline$R_{T}$ & \multicolumn{2}{c}{3,2300} & \multicolumn{2}{c}{3,2305} & \multicolumn{2}{c}{3,2656} \\
\hline \multicolumn{1}{c}{$\theta$} & $\varphi$ & $\theta$ & $\varphi$ & $\theta$ & $\varphi$ \\
\hline 1 & $0^{\circ}$ & $0^{\circ}$ & $0^{\circ}$ & $0^{\circ}$ & $0^{\circ}$ & $0^{\circ}$ \\
\hline 2 & $74,6^{\circ}$ & $0^{\circ}$ & $143,3^{\circ}$ & $0^{\circ}$ & $180^{\circ}$ & $0^{\circ}$ \\
\hline 3 & $74,6^{\circ}$ & $120^{\circ}$ & $143,3^{\circ}$ & $180^{\circ}$ & $90^{\circ}$ & $0^{\circ}$ \\
\hline 4 & $74,6^{\circ}$ & $240^{\circ}$ & $79,4^{\circ}$ & $48,7^{\circ}$ & $90^{\circ}$ & $72^{\circ}$ \\
\hline 5 & $130,2^{\circ}$ & $60^{\circ}$ & $79,4^{\circ}$ & $131,3^{\circ}$ & $90^{\circ}$ & $144^{\circ}$ \\
\hline 6 & $130,2^{\circ}$ & $180^{\circ}$ & $79,4^{\circ}$ & $228,7^{\circ}$ & $90^{\circ}$ & $216^{\circ}$ \\
\hline 7 & $130,2^{\circ}$ & $300^{\circ}$ & $79,4^{\circ}$ & $311,3^{\circ}$ & $90^{\circ}$ & $288^{\circ}$ \\
\hline
\end{tabular}

\section{GEOMETRIAS IDEAIS PARA OCTACOORDENAÇÃO}

Para espécies com oito átomos periféricos a geometria molecular ideal com valor de $R_{T}$ mais baixo (Tabela 5) e a anti prismática quadrada (simetria $D_{4 d}$ Figura $5 \mathrm{~A}$ ). Existem outras duas geometrias moleculares ideais com valores de $R_{T}$ próximos: a dodecaédrica (simetria $D_{2 \mathrm{~d}}$, Figura $5 \mathrm{~B}$ ) е a cúbica (simetria $O_{h}$, Figura 5 C).

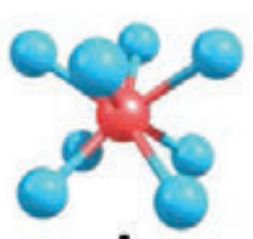

A

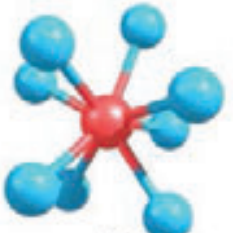

B

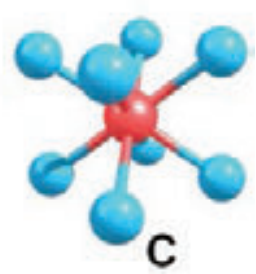

C
Figura 5 - Três geometrias de menor valor de $R_{T}$ para espécies do tipo $A B_{8}$
Tabela 5 - Valores de $R_{T}$ e coordenadas angulares usadas para os átomos periféricos referentes às três geometrias mais estáveis, para espécies do tipo $A B_{8}$

\begin{tabular}{|c|c|c|c|c|c|c|}
\hline & \multicolumn{2}{|c|}{$D_{4 d}$} & \multicolumn{2}{|c|}{$D_{2 d}$} & \multicolumn{2}{|c|}{$O_{h}$} \\
\hline$R_{T}$ & & & 5,2 & & 5,7 & \\
\hline & $\theta$ & $\varphi$ & $\theta$ & $\varphi$ & $\theta$ & $\varphi$ \\
\hline 1 & $57,1^{\circ}$ & $0^{\circ}$ & $37,3^{\circ}$ & $0^{\circ}$ & $54,7^{\circ}$ & $0^{\circ}$ \\
\hline 2 & $57,1^{\circ}$ & $90^{\circ}$ & $37,3^{\circ}$ & $180^{\circ}$ & $54,7^{\circ}$ & $90^{\circ}$ \\
\hline 3 & $57,1^{\circ}$ & $180^{\circ}$ & $71,4^{\circ}$ & $90^{\circ}$ & $54,7^{\circ}$ & $180^{\circ}$ \\
\hline 4 & $57,1^{\circ}$ & $270^{\circ}$ & $71,4^{\circ}$ & $270^{\circ}$ & $54,7^{\circ}$ & $270^{\circ}$ \\
\hline 5 & $122,9^{\circ}$ & $45^{\circ}$ & $108,6^{\circ}$ & $0^{\circ}$ & $125,3^{\circ}$ & $0^{\circ}$ \\
\hline 6 & $122,9^{\circ}$ & $135^{\circ}$ & $108,6^{\circ}$ & $180^{\circ}$ & $125,3^{\circ}$ & $90^{\circ}$ \\
\hline 7 & $122,9^{\circ}$ & $225^{\circ}$ & $142,7^{\circ}$ & $90^{\circ}$ & $125,3^{\circ}$ & $180^{\circ}$ \\
\hline 8 & $122,9^{\circ}$ & $315^{\circ}$ & $142,7^{\circ}$ & $270^{\circ}$ & $125,3^{\circ}$ & $270^{\circ}$ \\
\hline
\end{tabular}

\section{GEOMETRIAS IDEAIS PARA NONACOORDENAÇÃO}

Para espécies com nove átomos periféricos a geometria molecular ideal com valor de $R_{T}$ mais baixo (Tabela 6) é a prismática triangular de três faces "encapadas" (simetria $D_{3 h}$ Figura $6 \mathrm{~A}$ ). Existe outra geometria molecular ideal com um valor de $R_{T}$ próximo: a anti prismática quadrada de topo "encapado" (simetria $C_{4 v}$, Figura $6 \mathrm{~B}$ ).
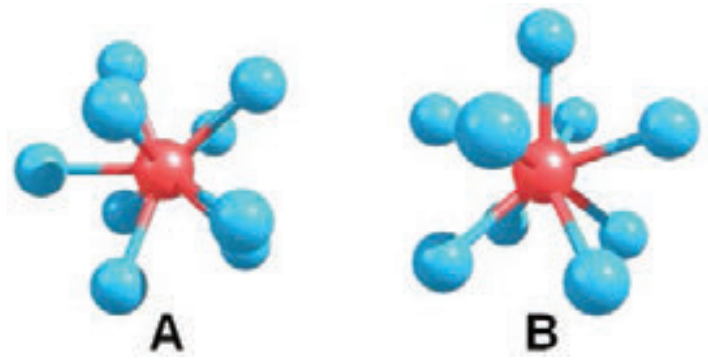

Figura 6 - Duas geometrias de menor valor de $R_{T}$ para espécies do tipo $A B_{9}$

Tabela 6 - Valores de $R_{T}$ e coordenadas angulares usadas para os átomos periféricos referentes às duas geometrias mais estáveis para espécies do tipo $A B$

\begin{tabular}{ccccc}
\hline & \multicolumn{2}{c}{$D_{3 h}$} & \multicolumn{2}{c}{$C_{4 v}$} \\
\hline$R_{T}$ & \multicolumn{2}{c}{8,1055} & \multicolumn{2}{c}{8,1175} \\
\hline 1 & $\theta$ & $\varphi$ & $0^{\circ}$ & $0^{\circ}$ \\
\hline 2 & $44,7^{\circ}$ & $120^{\circ}$ & $68,9^{\circ}$ & $0^{\circ}$ \\
\hline 3 & $44,7^{\circ}$ & $240^{\circ}$ & $68,9^{\circ}$ & $90^{\circ}$ \\
\hline 4 & $94,7^{\circ}$ & $60^{\circ}$ & $68,9^{\circ}$ & $180^{\circ}$ \\
\hline 5 & $90^{\circ}$ & $180^{\circ}$ & $68,9^{\circ}$ & $270^{\circ}$ \\
\hline 6 & $90^{\circ}$ & $300^{\circ}$ & $127^{\circ}$ & $45^{\circ}$ \\
\hline 7 & $135,3^{\circ}$ & $0^{\circ}$ & $127^{\circ}$ & $135^{\circ}$ \\
\hline 8 & $135,3^{\circ}$ & $120^{\circ}$ & $127^{\circ}$ & $225^{\circ}$ \\
\hline 9 & $135,3^{\circ}$ & $240^{\circ}$ & $127^{\circ}$ & $315^{\circ}$ \\
\hline
\end{tabular}




\section{CONCLUSÃO}

Os resultados obtidos estão de acordo com os publicados para as estruturas ideais [8], demonstrando-se assim, de forma prática e eficaz, que a utilização de meios informáticos facilmente acessíveis, bem como um conhecimento básico de Matemática e de Física permitem a obtenção de dados com interesse em Química.

\section{NOTAS}

Nota 1: É possível obter um ficheiro Excel já preparado para utilização, em http://www.spq.pt/boletim/suplementos.

Nota 2: Exemplo da constituição do ficheiro referente à geometria bipiramidal pentagonal, usando como átomo central o iodo e como átomos periféricos o hidrogénio, para uma hipotética molécula $\mathrm{IF}_{7}$ :

$\begin{array}{llllll}53 & 0 & 0 & 0\end{array}$

$90,0000 \quad 0,0000 \quad 1,0000$

$90,0000 \quad 0,0000-1,0000$

$9 \begin{array}{llll}1,0000 & 0,0000 & 0,0000\end{array}$

$\begin{array}{llll}9 & 0,3090 & 0,9511 & 0,0000\end{array}$

$9-0,8090 \quad 0,5878 \quad 0,0000$

$9-0,8090-0,5878 \quad 0,0000$

$90,3090-0,9511 \quad 0,0000$

\section{BIBLIOGRAFIA}

[1] Programa da disciplina de Física e Química A (níveis 1 e 2) $11 .^{\circ}$ ou $12 .^{\circ}$ anos: http://www.dgidc.min-edu.pt/ensinosecundario/ (Ministério da Educação e Ciência, acedido em 07-10-2013)

[2] A.R. Mahjoub, T. Drews, K. Seppelt, Angew. Chem. Int. Ed. Engl. 31 (1992) 1036-1039

[3] M.C. Favas e D.L. Kepert, Prog. Inorg. Chem. 28 (1981) 309-367

[4] D.L. Kepert, Prog. Inorg. Chem. 25 (1979) 41-144

[5] D.L. Kepert, Prog. Inorg. Chem. 24 (1978) 179-249

[6] M.G.B. Drew, Prog. Inorg. Chem. 23 (1977) 67-210

[7] http://www.chemcraftprog.com (acedido em 07-10-2013)

[8] D.L. Kepert, “Coordination Numbers and Geometries”, in G. Wilkinson, R.D. Gilland e J.A. McCleverty (eds.), "Comprehensive Coordination Chemistry", Vol. 1, Pergamon Press, Oxford (1987) 32-107

\section{Antiguidades Científicas}

\section{A CONTENDA LIEBIG-WÖHLER E O CONCEITO DE ISOMERISMO}

A revolução da Química nos finais do séc. XVIII e princípios do séc. XIX foi marcada por rupturas significativas. São disso exemplos o abandono da teoria do flogisto e a introdução da teória atómica de Dalton. Simultaneamente, com a criação de revistas especializadas e de academias, os resultados científicos passaram a ser divulgados de forma mais rápida e alargada o que, se por um lado, fomentou a troca de ideias, por outro, possibilitou também os desentendimentos públicos.

Nas primeiras décadas do séc. XIX, os químicos Justus Liebig (1803-1873) e Friedrich Wöhler (1800-1882) foram protagonistas de um famoso diferendo que haveria, no entanto, de ter um final feliz [1].
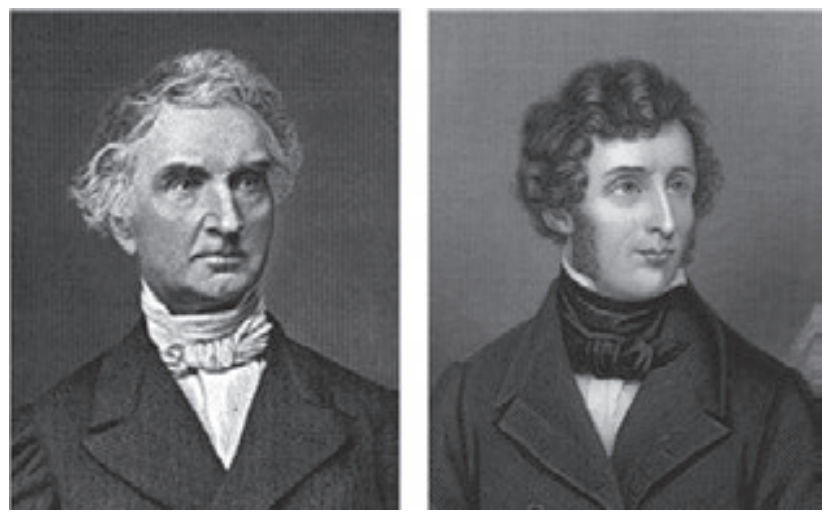

Justus Liebig (esquerda) e Friedrich Wöhler (direita)

Liebig, de origens modestas, começou como aprendiz de farmácia vindo a estudar Química nas Universidades de Bona e de Erlangen. Os bons resultados que obteve vale- ram-lhe uma bolsa de estudo para Paris, onde frequentou as aulas de prestigiados professores como Louis Jacques Thénard (1777-1857) e Louis Joseph Gay-Lussac (1778-1850). Foi no laboratório deste que se dedicou à análise orgânica, dispondo para o efeito das mais modernas técnicas da época. Interessou-se em particular pelo estudo dos derivados do ácido fulmínico $(\mathrm{H}-\mathrm{C}=\mathrm{N}-\mathrm{O})$, com os quais, em tempos - quando era um jovem aprendiz de farmácia - tinha provocado uma explosão [1]. Em 1824 chegou à fórmula do fulminato de prata, que publicou conjuntamente com Gay-Lussac [2].

Wöhler licenciou-se em Medicina em Heidelberg em 1823, apesar de a sua inclinação ser para a Química. Nesta disciplina teve Leopold Gmelin (1778-1853) como professor. Foi este que conseguiu que Wöhler fosse aceite no laboratório de Jacob Berzelius (1779-1848), em Estocolmo. Tal como Liebig em Paris, Wöhler pôde adquirir em Estocolmo uma vasta experiência em Química Analítica. No laboratório de Berzelius analisou o cianato de prata, concluindo que se tratava de um sal de um ácido desconhecido, o ácido ciânico $(\mathrm{H}-\mathrm{O}-\mathrm{C} \equiv \mathrm{N})$. Os resultados desta análise quantitativa eram surpreendentemente idênticos aos que Liebig tinha obtido para o fulminato de prata [3].

Liebig, pessoa honesta e bem formada, era todavia detentor de um temperamento algo agressivo e impetuoso que o levou de imediato a acusar Wöhler de não saber fazer análises. Liebig acabaria por analisar uma amostra de cianato de prata que o próprio Wöhler lhe enviou. Os resultados obtidos não deixaram então margem para dúvida: eram 
iguais aos de Wöhler [4]. Deste modo, Liebig viu-se obrigado a admitir publicamente a incorrecção de ter posto em causa as capacidades do colega como químico analítico. Foi nestas circunstâncias que acabou por nascer entre os dois químicos uma amizade que duraria até ao fim das suas vidas, bem como uma frutuosa colaboração científica [6].

Os achados de Wöhler e de Liebig criaram um embaraço à época, pois era entendimento geral que dois compostos com propriedades distintas deveriam possuir composições químicas diferentes. A solução para o problema veio de Berzelius, o mestre sueco de Wöhler e um dos maiores químicos do século [6]. De resto, outros factos contribuíram para a racionalização do problema. Em 1825, ao investigar o material oleoso que se obtinha da queima da gordura de baleia para obtenção do gás de iluminação, Michael Faraday (1791-1867), isolou dois produtos: um líquido, o benzeno, e o outro gasoso, com uma composição percentual idêntica à do gás oleificante (etileno, $\mathrm{C}_{2} \mathrm{H}_{4}$ ), apesar da diferente densidade. Hoje sabe-se que esse gás é o isobutileno $\left(\mathrm{C}_{4} \mathrm{H}_{8}\right)$. Em 1822, Berzelius já tinha observado existirem dois óxidos de estanho diferentes com a mesma composição e, em 1828, a ureia que Wöhler sintetizou mostrou ter a mesma composição do cianato de amónio. Em 1823, Eilhard Mitscherlich (1794-1863) observou que os cristais de enxofre apresentavam formas (monoclínica ou rômbica) que variavam de acordo com o modo de preparação. Também se sabia que a aragonite e a calcite eram duas formas cristalinas do carbonato de cálcio e que tanto a pirite como a marcassite eram $\mathrm{FeS}_{2}$. Sabia-se também que o diamante e a grafite eram ambos carbono e por volta de 1826 foi o próprio Berzelius quem descobriu que o ácido tartárico e o ácido racémico (racemato do ácido tartárico) tinham a mesma composição, apesar da diferença nalgumas das suas propriedades [1,6]. Assim, em 1831, Berzelius propôs o conceito de isomerismo (da palavra grega que significa “partes iguais”), de acordo com o qual compostos isoméricos teriam a mesma composição, contendo igual número absoluto e relativo de átomos mas apresentando propriedades distintas [7]. Disso eram exemplos o fulminato de prata e o cianato de prata. Por sua vez, o gás oleificante e o isobutileno não seriam isómeros já que possuíam o mesmo número relativo de átomos.

Para Berzelius, o isomerismo provinha da possibilidade de os átomos poderem ligar-se de diferentes modos para formar moléculas, o que era perfeitamente concebível à luz da teoria atómica de Dalton. Esta explicação foi bem aceite e - apesar de na época o conceito de massa molar ser ainda vago - o termo isómero adquiriu gradualmente o seu significado actual. Em 1841 Berzelius haveria também de introduzir o conceito de alotropia para as diferentes variedades de um elemento, enquanto que o conceito de polimorfismo era aplicado a compostos que ocorrem em mais do que uma forma cristalina [8].

Como observação final assinale-se que Gay-Lussac tinha já notado em 1814 que a composição percentual do ácido acético e da celulose são idênticas e que "o arranjo dos átomos num composto tem a maior influência no seu carácter neutro, ácido ou alcalino”, abrindo assim, implicitamente, o caminho para a descoberta do isomerismo $[9,10]$.

\section{NOTAS COMPLEMENTARES}

Wöhler destacou-se inicialmente em estudos da quinona e da hidroquinona, importantes para o desenvolvimento da Química Orgânica, mas a partir de 1839 centrou-se fundamentalmente na Química Inorgânica. Nesta área deu, entre outros, contributos relevantes para o conhecimento da química do boro, do silício e do fósforo, tendo ainda isolado o berílio e o alumínio [1]. A sua longa carreira académica iniciou-se em 1825 na Escola Politécnica de Berlim, mudando-se posteriormente para a Universidade de Göttingen, onde permaneceu até à morte. Em 1834 foi eleito membro da Academia Real Sueca das Ciências. O seu nome ficou para sempre associado à descoberta da síntese da ureia (1828) pela contribuição decisiva que esta teve para a rejeição da doutrina do vitalismo [11,12].

Leibig teve igualmente uma carreira académica brilhante, iniciada em 1824 na Universidade de Giessen (sob recomendação do famoso navegador Alexander von Humbolt), onde fundou uma escola de ensino e de investigação de grande reputação. Vinte e oito anos depois aceitou um lugar na Universidade de Munique, que ocupou até ao fim da vida. É considerado o “pai” da Química Agrícola, tendo efectuado, entre outros, estudos sobre o nitrogénio como elemento nutriente das plantas e de desenvolvimento de fertilizantes artificiais. Destacou-se também na Química Biológica com a criação de um método de produção de extracto de carne, o qual foi comercializado a partir de 1865. Foi editor da revista Annalen der Chemie und Pharmacie e em 1845 recebeu o título de barão. O seu congenial "mau-feitio” fê-lo incompatibilizar-se com vários colegas, entre os quais Louis Pasteur e Jacob Berzelius.

Da grande amizade e da colaboração científica entre Justus Leibig e Friedrich Wöhler ficou um legado de mais de mil cartas que trocaram entre si [1].

\section{REFERÊNCIAS}

[1] S. Esteban, J. Chem. Educ. 85 (2008) 1201-1203

[2] J.L.Gay-Lussac, J. Liebig, Ann. Chim. Phys. 25 (1824) 285-311

[3] F. Wöhler, Poggendorffs Ann. Phys. Chem. 1 (1824) 117124

[4] J. Liebig, Schweiggers Jahrbuch der Chemie und Physik. 18 (1826) 380

[5] E. Berl, J. Chem. Educ. 15 (1938) 553-562

[6] R. Winderlich, J. Chem. Educ. 25 (1948) 501-505

[7] J. J. Berzelius, Jahresberichte über die Fortschritte der Physischen Wissenschaften 10 (1831) 82; 11 (1832) 44

[8] A. J. Ihde, “The Development of Modern Chemistry”, Harper \& Row, Nova Iorque, 1964

[9] F. Kurzer, J. Chem. Educ. 77 (2000) 851-857

[10] J.L. Gay-Lussac, Ann. Chim. 41 (1814) 149

[11] T. O. Lipman, J. Chem. Educ. 41 (1964) 452-458

[12] W.H. Warren, J. Chem. Educ. 5 (1928) 1539-1553

João Paulo André

(jandre@quimica.uminho.pt) 
